\title{
Investigation of the effect of passive vortex inserts of different geometrical shapes on the vibrations reduction efficiency in pipelines with two-phase flow
}

\author{
Vladimir I. Velkin*, Sergey E. Shcheklein, Hossain Ismail, A. Nikitin, and G.Chikansev \\ Ural Federal University named after the first President of Russia B. N. Yeltsin \\ 19 Mira str., Yekaterinburg 620002, Russia
}

\begin{abstract}
This paper describes the experimental test bench used in coolant flow research with application of adjusted optical laser system. It is based on the technology of pulsed particle visualization of micro tracers, i.e. Particle Image Velocimetry (PIV system) that allows for the analysis of the microstructure of the hydrodynamic liquid flow passed through the vortex inserts. Optical measurement (PIV-method) of hydro- and aerodynamic characteristics of the coolant flow allowed obtaining the velocity distribution in straight and various curved sections of the pipeline in order to reliably define the vortex formation condition and the occurrence of the pressure gradient on the outer and inner walls of the curved sections. This gives us the opportunity to verify the calculation models describing the problem of passive control (decrease) of the displacement of pipelines of power equipment, and thus to improve the reliability and safe operation of the system in general.
\end{abstract}

\section{Introduction}

Reliable operation of power equipment of thermal and nuclear power plants is connected with the solution to the problem of reducing the vibrations of pipelines transporting twophase flows. Significant levels of vibration, particularly in pipelines with two-phase flow condensation, lead to a sharp increase in the cost of their maintenance, reduction of reliability of the energy system, and in critical cases causes the depressurization of the coolant flow paths due to fatigue failure of the pipeline metal [1]. One of the directions in solving the problem of reducing vibration in pipelines is carrying out research towards optimizing the geometry of passive devices that affect the hydrodynamics of a two-phase flow [2]. The two-phase flow in the pipelines of the power equipment is realized in the form of various hydrodynamic structures and manifests itself in the form of pulsations of the main parameters of the stream. To reduce or eliminate this phenomenon, various kinds of active and passive devices are used that change the hydrodynamic flow structure [3].

\footnotetext{
*Corresponding author: v.i.velkin@urfu.ru
} 


\section{Experimental test bench}

For this research purpose an experimental test bench was designed that enabled carrying out forced circulation of the mixture consisting of coolant and injected air to form various modes of two-phase flow (Fig. 1).

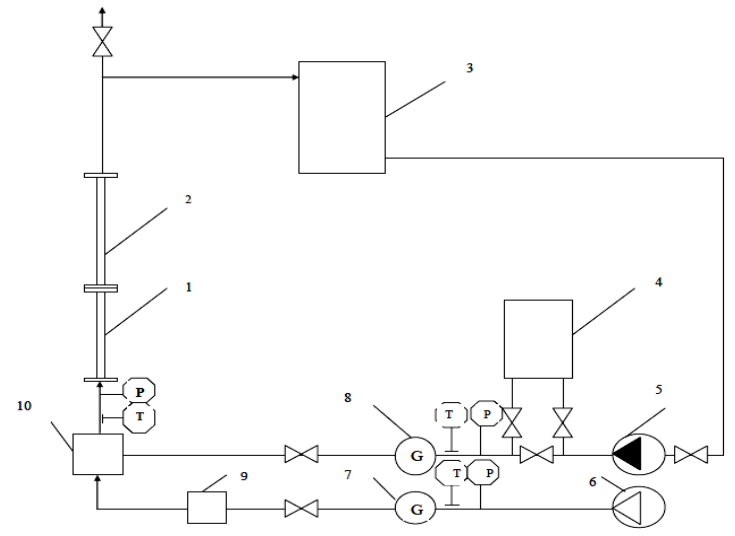

Fig.1. The schematic diagram of the experimental test bench 1 - vortex insertion; 2 - test section of the channel; 3 - water tank; 4 - control panel; 5 - pump; 6 compressor; 7 - air flowmeter; 8 - water flowmeter;9 damper; 10- blending unit.
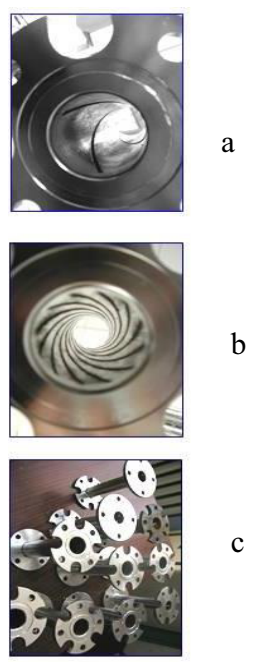

Fig.2. Photos of vortex inserts: a) with three grooves; b) with twelve grooves; c) - a set of investigated samples of vortex inserts with different internalsurface geometries.

Effectiveness analysis of various kinds of vortex inserts relating to passive means of reducing the vibration of pipelines has shown that the use of inserts with coiled surface grooves is quite promising and has a certain advantage over the other devices [4]. In particular, they have less hydraulic resistance and can be installed in series along the length of the pipeline before entering the turn. Figure 2 shows vortex inserts with different internal surface geometries.

Investigations of the twisting effect in two-phase flow with benefit of vortex inserts with involute grooves were carried out on a hydrodynamic test bench with an air-water flow circulating in a closed loop of $45 \mathrm{~mm}$-diameter pipe with transparent Plexiglas sections for flow visualization [5].

\section{Comparison of vortex inserts}

We take the ratio between the kinetic energy of the swirling part of the flow ${ }_{\mathrm{B}}^{\mathrm{B}} \mathrm{d}$ the energy of the translational (axial) flow at the outlet of the swirler as the energy characteristic of the twist intensity.

$$
\varepsilon=(\operatorname{tg} \varphi)^{2} \mathrm{nf} / \mathrm{F}_{0} ; \operatorname{tg} \varphi=\mathrm{U}_{\varphi} / \mathrm{U}_{0}
$$

Where $\mathrm{n}-$ is the number of swirl grooves; $\mathrm{f}$ and $\mathrm{F}_{0}$ - are the values of the total crosssectional area of the grooves and the cylindrical channel; $\mathrm{U} \varphi$ and $\mathrm{U}_{0}$ - are the tangential and axial flow velocities.

According to the Darcy-Weisbach equation for a single-phase flow the hydraulic characteristics of the vortex inserts can be figured out from the pressure drop measurements: 


$$
\xi=2(\mathrm{~d} / \mathrm{L})\left(\Delta \mathrm{p} / \rho \mathrm{U}_{\mathrm{o}}^{2}\right)
$$

where $\xi$ - is the coefficient of hydraulic resistance (CHR) of the insert; $d$ and L - are the hydraulic diameter and length of the insert.

\section{Study of the vortex inserts effectiveness using the PIV method}

Theoretical and experimental data obtained during the experiments on the test bench need to be analyzed with the help of more sophisticated methods and tools for studying micro flows. To verify the results of mathematical modeling within the framework of this task, a digital image velocimetry (PIV) method was applied. It makes it possible to measure the velocity fields in the channels within the submillimeter range with a resolution of the vector field up to 2-5 $\mu \mathrm{m} /$ vector in the cross section just a few $\mu \mathrm{m}$ thick. The determination of the displacement is based on the application of correlation methods to the tracer pictures using regular partitioning into elementary regions.

For the experiments, we used the "Polis" measuring system, which made it possible to measure the spatial distributions of two and three components of the instantaneous flow velocity, to calculate the spatial derivatives of the velocity, and to calculate a whole set of mixed statistical moments (up to the fourth order) [6]. The scheme of the used flowmeasuring structure is shown in Fig. 3.

Recording of the reflected optical pulses is carried out by the cross-correlation camera, which is designed for double adjustment of trace patterns in the stream and can work both in the two-frame and single-frame modes. The test bench is equipped with a tracer device (High powered Fog generator Martin Magnum 1800). The facility allows the study of flow regimes at Reynolds number $10^{4}-10^{6}$ that ensures the applicability of the results obtained for the analysis of aerodynamics of real-scale installations [7, 8]. To measure the velocity fields in the concerned model sections we applied the PIV-system "Polis" developed at the Institute of Thermophysics SB RAS [9,10]. Figure 4 shows the photo visualization of the fluid flow swirling $(\mathrm{f}=0)$ after transition through the vortex insert.

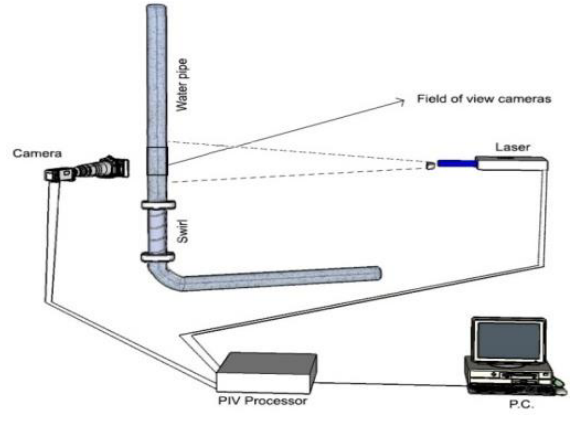

Fig.3. The scheme of the flow-measuring coolant flow after transition of the structure "Polis" as part of a laser installation, receiving chamber, synchronizer, and PCimage processing unit.

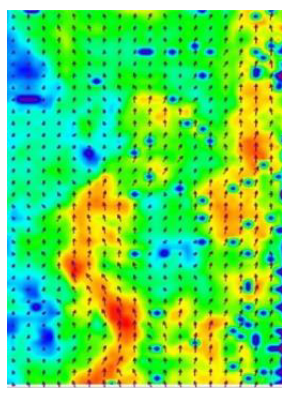

a )

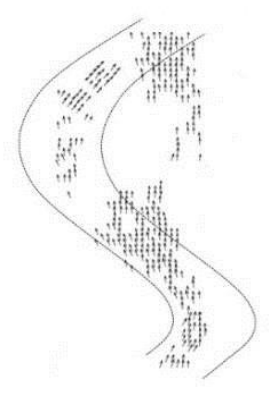

b)
Fig.4. a) Color photo visualization of the fluid flow swirling in the absence of gas after transition through the vortex insert; $b$ ) the tracer of the velocity vector of the swirl insert at the section $\mathrm{L}=0-15 \mathrm{~cm}$.

The different colors of the vectors in Fig. 6a correspond to different velocities. After a small image processing using the application program, we get a picture of the velocity 
vectors of a swirling fluid flow (Fig. 6b). The presented flow vector of the flow velocity vectors on the straight section of the pipeline demonstrates the distribution of the velocity vectors after leaving the swirl insert and shows the dispersion of the pressure gradient of the aqueous medium from the outer wall throughout the pipeline.

\section{Conclusions}

1. We designed the vortex insertion devices for passive control of pipeline vibrations which allow reducing the vibration displacement level of pipelines with two-phase flows by factor of 1.5-2 at a relatively low hydraulic resistance.

2. To verify the results of the simulation, an experimental test bench was designed and an optical laser system based on the pulsed imaging technology of micro trackers particles (PIV-system) was adjusted for measurements.

3. The system of optical measurement of hydrodynamic characteristics of the coolant flow (PIV-method) will allow obtaining velocity distributions in straight and various curved sections of the pipeline, as well as solving problems of passive control (reduction) of vibration displacement of pipelines of power equipment, and increasing reliability and safety of operation in general.

\section{References}

1. V.I. Baranenko, S.G. Oleinik, O.A. Belyakov, R.S. Istomin, A.V. Kumov, Fourth International Scientific and Technical Conference" Ensuring the Safety of NPPs with $V V R$, Podolsk, FSUE OKB" GIDROPRESS. (2005) [in Russian]

2. E.D. Fedorovich, B.S. Fokin, A.F. Axelrod \& others, M: Energoatomizdat (1989). 168

3. M.Ya. Belenky., M.A Gotovsky., B.S. Fokin, Teploenergetika. 3, 41 (1996)

4. V.I. Kasina, A.I. Leontiev, Heat-power Engineering 3, 40 (2005)

5. V.I. Velkin, D.S.Komoza, A.Yu. Krutikov, V.V.Khnykina, News of Higher Educational Establishments "Nuclear Energy", 1, 4 (2009) [in Russian]

6. E.K. Akhmetbekov, A.V. Bilsky, D.M. Markovich, A.A. Maslov, P.A. Polivanov, I.S. Tsyryulnikov, M.I. Yaroslavtsev, Thermophys. Aeromech. 16, 343 (2009)

7. A.A. Maslov, S.G. Mironov, Izv. RAS. FGM. 6, 155 (1996)

8. F. Scarano. Overwiew of PIV in supersonic flows. Particle Image Velocimetry / Ed. By A. Schroeder, C.E. Willert. (Springer-Verlag, Berlin, Heidelberg, 2008)

9. M.P. Tokarev, D.M. Markovich, A.V. Bilsky, Computational technologies. 12, 109 (2007)

10. S.V. Alekseenko, A.V. Bilsky, D.M. Markovich, Devices and experimental technique. 47, 703 (2004) 